\title{
THE MEASUREMENT OF BLOOD PRESSURE IN OESOPHAGEAL VARICES
}

\author{
BY \\ PHILIP ALLISON \\ From the General Infirmary at Leeds \\ (RECEIVED FOR PUBLICATION JULY 4, 1951)
}

The surgical treatment of portal hypertension surely needs objective assessment. The various intra- and extra-hepatic disorders which produce portal hypertension are not amenable to surgical correction, and efforts have been confined to the alleviation of symptoms, the two main ones being haematemesis and recurrent ascites. It is not intended here to describe or criticize the many ingenious ways in which surgeons have assailed the problems, but it would be fair to say that discretion has not always kept pace with enthusiasm and that, as most of the operations are formidable, much harm has been done without any compensatory promise of success. There has also been a tendency to claim good results without a sufficiently prolonged appraisal of the post-operative progress of the patient in relation to the known natural history of the disease. If a patient with portal hypertension has persistent and recurrent ascites an operation which leaves him free from ascites might be accepted as successful ; but the problem is not even as simple as this, for in one patient a laparotomy alone may suffice ; in another a splenectomy may be enough ; and in a third an operation of venous anastomosis may work when all else has failed even though the anastomosis does not stay patent. A poor patient who comes into hospital, has his abdomen tapped, and is given a good diet and blood transfusions may improve from these rather than from anything done at operation. The tendency to ascites undergoes inexplicable remissions in the absence of surgery. If the results are to be assessed in terms of clinical improvement of the specific symptom for which the operation is performed, a surgeon must operate on very many patients and wait many years before he can present reasonable figures. If at the end of this time the operation is found wanting, those who have died have done so in vain, and those who have survived have suffered to no purpose. As few surgeons are content to wait so long for assessment, the situation is made much worse by the operation being taken up by others who may be less skilled but equally eager.

What has been said of ascites is even more applicable to haematemesis, for a patient may have natural intervals of years between the attacks. A patient with portal hypertension who vomits blood does so from oesophageal or gastric varices. There has been the most noticeable lack of reports in the literature of operations done for varices and controlled even by oesophagoscopic inspection of the varices. Radiographic evidence is unreliable, for varices may be demonstrated or not in the same patient without any operation being performed. Unless they are very gross, they may easily be missed. But direct inspection through the oesophagoscope is absolutely reliable and should never be omitted. 
Pressures taken in the portal vein on the operation table just before and just after a venous anastomosis may, or may not, show a fall in portal pressure, but this does not give any indication of the pressures three weeks or three months later when the anastomosis may have beccme occluded. Oesophagoscopic observation of the varices may suggest that they are less distended than before operation, but this is most unreliable if the time interval is as long as it should be. It was these considerations that pointed to the necessity for an objective test for portal pressures which could be repeated at intervals, and it was the frequent inspection of the oesophageal veins which suggested them as suitable for such a test. It seems more important to know the pressures in the actual veins that bleed, and how these can be affected, than to know the pressures in the portal vein itself. If any operation that is devised for portal hypertension with haematemesis from varices can be judged by such an objective method, its effectiveness or otherwise will be apparent immediately. This is still valid even though the disease causing the portal hypertension may progress and undo the good that has been achieved. The surgical attack is not directed against the disease, but against the local manifestation which is responsible for haematemesis.

\section{INSTRUMENTS}

The adult medium-sized Negus oesophagoscope is used (Fig. 1). This instrument is $50 \mathrm{~cm}$. long, and has an oval lumen 15 by $13 \mathrm{~mm}$., with an anterior lip at the advancing
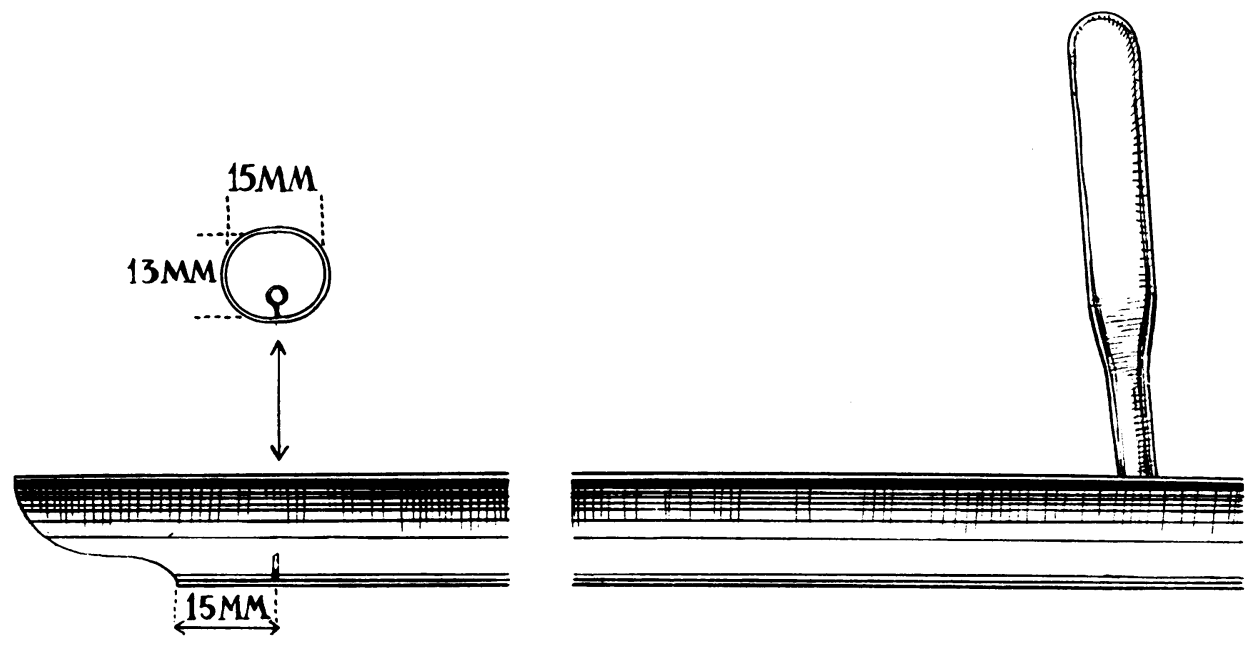

FIG. 1.

end projecting $25 \mathrm{~mm}$. beyond the posterior lip. For this particular use it has been modified by welding into the posterior wall a small peep sight $2 \mathrm{~mm}$. in diameter which projects into the lumen of the oesophagoscope on a small pedicle $2 \mathrm{~mm}$. long. This is placed $15 \mathrm{~mm}$. from the posterior lip and is used to support and steady the needle which is used to puncture the vein. 
The needle (Fig. 2) is $65 \mathrm{~mm}$. long with an internal bore of $0.9 \mathrm{~mm}$. At a point $45 \mathrm{~mm}$. from its base and $20 \mathrm{~mm}$. from its tip it is bent at an angle of 14 degrees. There is a short bevel on the needle, and a lateral hole $4 \mathrm{~mm}$. from the tip on the opposite side to the bevel, so that if the end of the needle is against the vein wall the pressure will be transmitted through the lateral hole. The base of the needle is welded on to the end of a Chevalier Jackson bronchoscopic suction tube, the join being smoothed down so that there is no obstruction to vision.

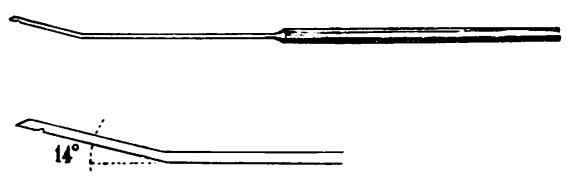

FIG. 2.

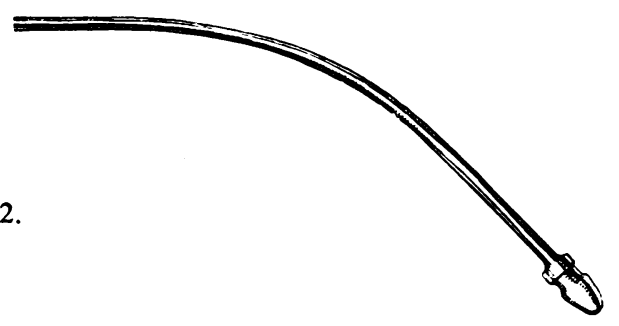

The pressures have been recorded by a Tyberg Hansen manometer.

\section{METHOD}

The patient is given $\frac{1}{4}$ gr. morphine and $1 / 100 \mathrm{gr}$. atropine. The throat is anaesthetized with $2 \%$ amethocaine hydrochloride, and then an intravenous anaesthetic of curare and pentothal is given until respiration is quiet and the cough reflex gone. The oesophagoscope is passed and the varices inspected. A suitable varix is selected on the posterior wall of the oesophagus and placed in the field just over and somewhat to the right of the peep sight. The needle is connected to the Hansen manometer and a constant slight drip of heparin solution maintained after all air bubbles have been expelled. Zero and calibrating tracings are taken, and then the needle is passed through the oesophagoscope and through the peep sight at the lower end. The bend on the needle allows the point to be kept in view beyond the peep sight, and the latter acts as a steady support. The varix is then pierced with the needle, and, after blood has been drawn back into a syringe attached to a side tube so that there is no doubt about the needle being in the lumen, a pressure reading is taken. When the needle is withdrawn the oesophagoscope is passed over the puncture to compress it for a short time while the calibration and zero readings are repeated on the manometer. If necessary a second varix may be punctured at a lower level with a different calibration level on the manometer to check the accuracy of the reading. A little oozing may take place from the site of puncture, but this has never been significant. No untoward complications have been experienced. The pressures have been arbitrarily measured from the top of the table on which the patient lies. 